\title{
Pieu sous charge latérale cyclique : incertitudes sur les mesures
}

\section{F. ROSQUOËT}

Laboratoire des technologies innovantes Équipe Phénomènes de transferts et construction durable IUT d'Amiens

\section{THOREL, J. GARNIER}

Laboratoire central des Ponts et Chaussées de Nantes Route de Bouaye BP 4129 44341 Bouguenais Cedex
L'effet des cycles de chargement horizontal en tête de pieu sur l'interaction sol-pieu est étudié sur modèles réduits centrifugés. L'évolution avec le nombre de cycles du déplacement au point d'application de la charge et du moment maximum est analysée avec une interpolation logarithmique en incluant un calcul d'incertitude.

Mots-clés : pieux, charge latérale cyclique, incertitude, centrifugeuse.

\section{Pile under lateral cyclic load: uncertainty of a measurement}




\section{Introduction}

Le chargement latéral cyclique des pieux est généralement le résultat des sollicitations mécaniques engendrées par les vagues, le vent sur des structures offshore, l'accostage ou l'amarrage de bateaux, les surcharges variables ou les dilatations thermiques. L'étude paramétrique sur des ouvrages géotechniques réels, tels que les fondations profondes, est relativement difficile. C'est pourquoi on fait souvent appel à des modèles réduits centrifugés (Garnier, 1995), méthode permettant une étude paramétrique et une mise en ceuvre aisée, tout en ayant une bonne maîtrise des conditions limites (Remaud, 1999). L'étude expérimentale sur un pieu modèle instrumenté souple permet d'acquérir un nombre important de données (Rosquoët, 2004) : évolution du profil de moment fléchissant et du déplacement en tête du pieu, en fonction de la charge appliquée. Ces

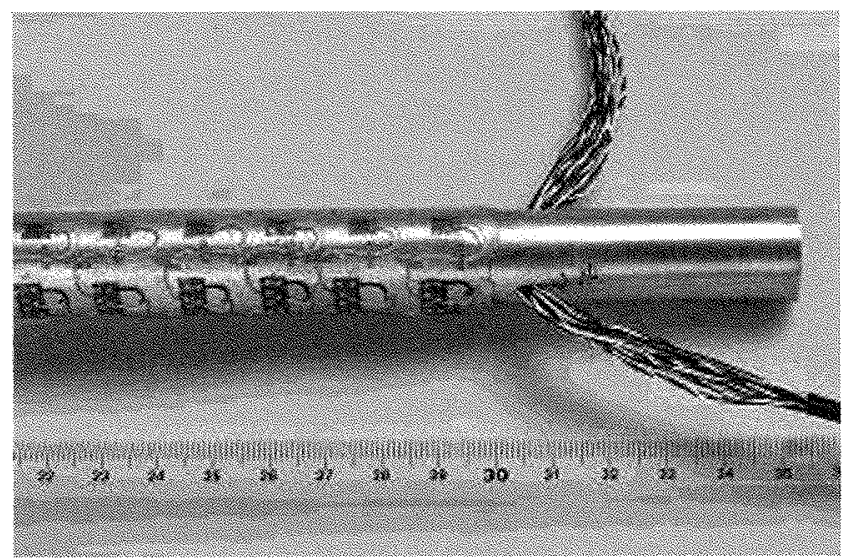

mesures, effectuées avec soin malgré l'environnement contraignant de la macrogravité, sont toujours entachées d'incertitudes.

Le déplacement et le moment maximal relatif (aux valeurs du premier cycle), exprimés en fonction du nombre de cycles, peuvent être interpolés par une équation logarithmique. Après avoir déterminé les incertitudes sur les mesures de déplacement, de force et de moment, nous nous intéressons à l'influence de ces incertitudes sur l'interpolation logarithmique.

\section{Dispositif expérimental}

Le pieu isolé (Figs. 1 et 2) est mis en place à $1 \times g$ par battage, dans un massif de sable de Fontainebleau NE34 reconstitué par pluviation dans l'air (Rosquoët, 2004).

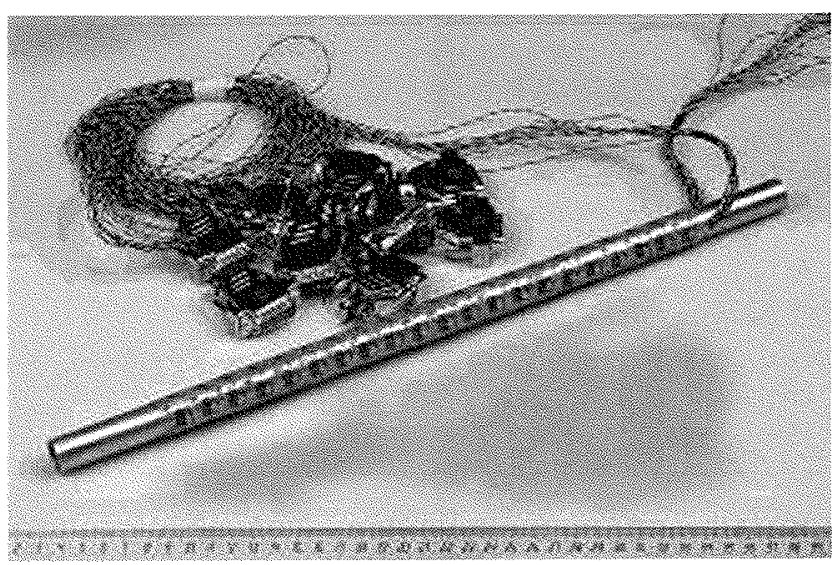

FIG. 1 Pieu instrumenté de $18 \mathrm{~mm}$ de diamètre et détail des jauges collées à sa surface pour la mesure des moments. Le câblage passe à l'intérieur du pieu.

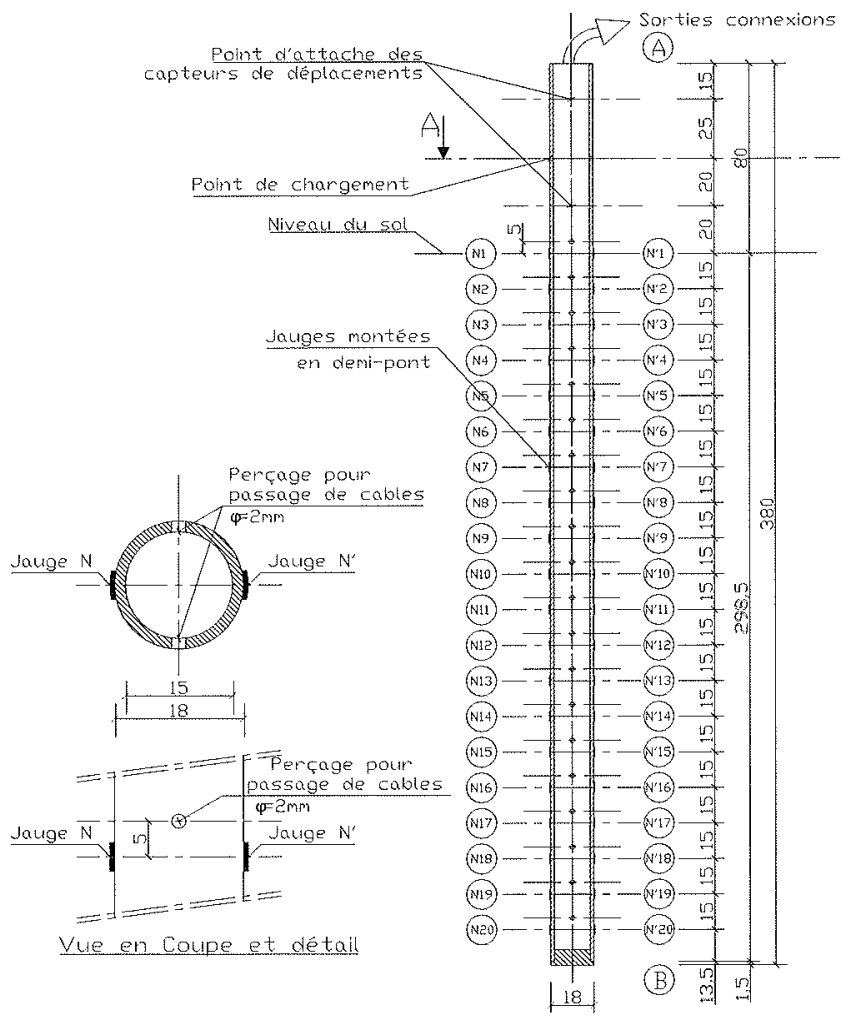

Fic.2 Schéma du pieu instrumenté.
Sous $40 \times g$ d'accélération centrifuge, un servo-vérin horizontal (Thoret et al., 2008) placé sur un bâti rigide fixé sur le conteneur d'essai permet d'appliquer le chargement à $40 \mathrm{~mm}$ au-dessus du sol. L'effort est mesuré à l'aide d'un capteur de force, d'une capacité maximale de 500 daN. Pour le chargement unidirectionnel non alterné (traction uniquement) un câble assure la liaison entre le capteur et le pieu modèle de longueur $380 \mathrm{~mm}$ (Fig. 3).

Les deux capteurs de déplacement (d'une course de $100 \mathrm{~mm}$ ) sont fixés par l'intermédiaire d'une rotule sur le pieu modèle (pour éviter d'introduire des moments de flexion parasites lors des essais de chargement) et sont placés respectivement à $20 \mathrm{~mm}$ et de $65 \mathrm{~mm}$ du sol (Fig. 4).

En supposant que le pieu ne se déforme pas en dehors du sol (uniquement rotation et translation), ces deux capteurs permettent de déterminer le déplacement latéral ( $\mathrm{y}$ » du pieu au point d'application de la charge, transmise par le câble. $y_{c 1}$ et $y_{c 2}$ sont les mesures respectivement obtenues par les capteurs de déplacement 1 et 2. " y ) est calculé en appliquant le théorème de Thalès :

$$
y=y_{c 1}+\left(y_{c 2}-y_{c 1}\right) \frac{20}{45}
$$

Les informations collectées par les différents capteurs au cours d'une expérimentation transitent par l'intermédiaire d'une chaîne d'acquisition qui transforme des valeurs électriques en valeurs physiques. 

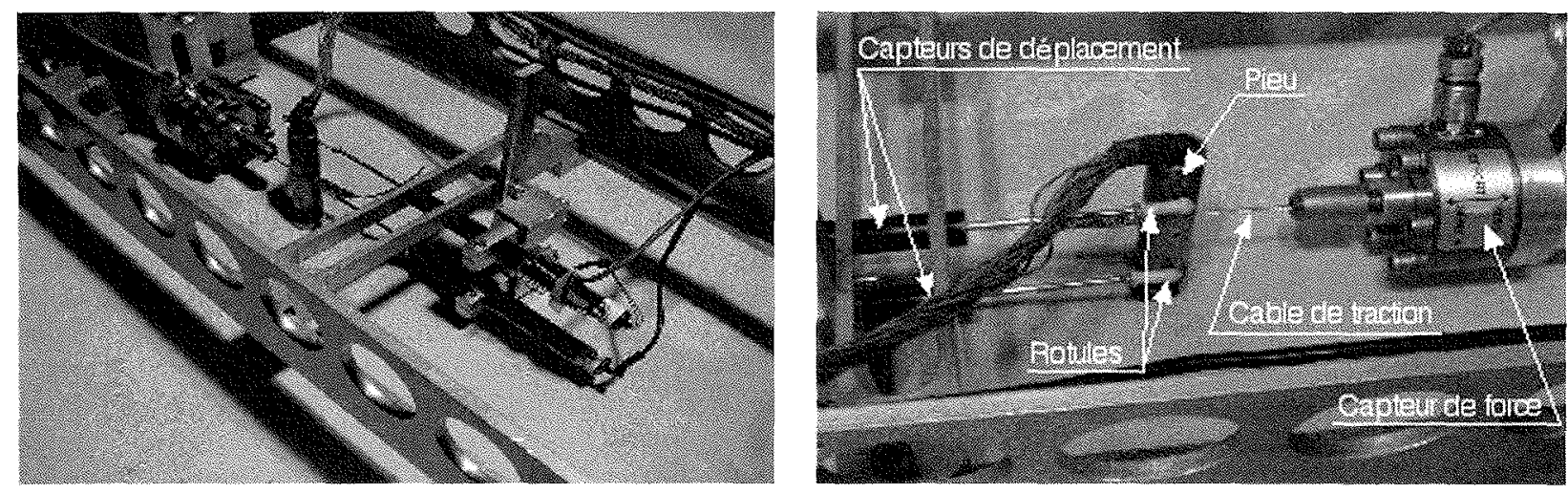

FG. 3 Dispositif de chargement : pieu installé dans un massif de sable soumis à un chargement horizontal.

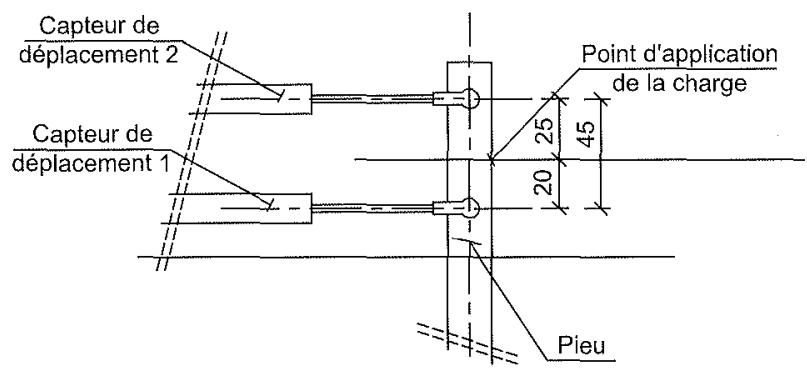

rc 4 Position des capteurs de déplacement.

Les mesures physiques sont entachées d'incertitudes généralement liées à la précision des appareils utilisés. On note également que la précision d'usinage des différents éléments mécaniques, ou bien encore la résolution de la chaîne d'acquisition utilisée, doivent être prises en compte pour quantifier le plus précisément possible les incertitudes que l'on commet lors de la mesure du déplacement, de la force ou des moments. Cependant, il faut bien garder à l'esprit que toutes les erreurs liées à ces expérimentations ne peuvent pas toujours être quantifiées. Le chargement cyclique est caractérisé par la charge maximale $F$ et l'amplitude de variation de charge DF. Dans le cas d'un chargement cyclique de type non alterné, l'effort est toujours appliqué dans le même sens, la charge variant entre F et F-DF:

Le pieu est instrumenté par 20 niveaux de jauges de déformation, permettant d'identifier le profil des moments fléchissants. En supposant que le comportement du matériau est élastique linéaire, il existe une relation entre la charge appliquée Q à l'extrémité de la poutre et le signal électrique $u_{i}$ mesuré sur chaque demi-pont de jauge. A chaque niveau $z_{i^{\prime}}$ en prenant en compte la rigidité à la flexion du pieu et le signal électrique obtenu par la déformation d'une jauge, le moment s'écrit :

$$
M\left(z_{i}\right)=4 \frac{E I}{B} \frac{u_{i}}{u} 10^{-3}
$$

où E est le module d'Young du matériau; I, le moment d'inertie ; $u_{i^{\prime}}$ la tension de mesure ; $u$, la tension d'alimentation ; $\Omega$, le facteur de jauge et $\mathrm{B}$ le diamètre du pieu.

\section{3 \\ Hypothèses}

Plusieurs hypothèses sont nécessaires à la conduite des calculs d'incertitude présentés par la suite. On suppose que les mesures ne sont soumises qu'à des incertitudes aléatoires et que la distribution limite engendrée par un grand nombre de valeurs mesurées $($ ( $X)$ tend vers un maximum unique, correspondant à la valeur moyenne ( $X)$, tandis que les autres valeurs se répartissent régulièrement de part et d'autre de cette valeur maximale suivant ainsi une loi normale. Lorsqu'une mesure suit une loi normale, la probabilité qu'une mesure $x$ se situe dans un intervalle $[a, b]$ peut s'écrire, en introduisant l'écart-type $\sigma$, sous la forme suivante :

$$
P(x \in[a, b])=(1 / \sigma \sqrt{2 \pi}) \int_{a}^{b} e^{-\frac{(x-x)^{2}}{2 \sigma^{2}}} d x
$$

La probabilité qu'une mesure $\mathrm{x}$ quelconque soit comprise entre la valeur maximale et plus ou moins t fois l'écart-type revient à calculer l'intégrale de l'équation (3) en remplaçant les bornes a et b par et $\mathrm{X}$ - to et $\mathrm{X}+\mathrm{t} \sigma$. Si on pose $\mathrm{z}=\frac{(\mathrm{X}-\mathrm{X})}{\sigma}$ ce changement de variable (avec $d x=\sigma d z$ ), permet d'écrire :

$$
P(x \in[X-t \sigma, X+t \sigma])=P(z \in[-t, t])=\frac{1}{\sqrt{2 \pi}} \int_{-t}^{t} e^{-\frac{z^{2}}{2}} d z
$$

L'équation (4) connue sous le nom « intégrale de l'erreur normale » permet de calculer la probabilité qu'une mesure $\mathrm{x}=\mathrm{x}_{\text {mesuré }} \pm \delta \mathrm{x}$ tombe à moins d'un écart type (Taylor, 1982). Cette probabilité $\mathrm{P}(\mathrm{z} \in[-1,1])$ est égale à $68 \%$. Le calcul montre que, pour une mesure égale à $3 \sigma / 2$, la probabilité est de $87 \%$ alors qu'elle passe à $95,4 \%$ pour $2 \sigma$. Au vu de ces résultats, il est généralement de coutume de prendre l'incertitude associée à une mesure comme étant égale à l'écarttype $(\delta \mathrm{x}=\sigma)$.

Dans le cas de calcul de paramètres basés sur $\mathrm{N}$ mesures indépendantes $a_{i}$, si les incertitudes sont indépendantes et aléatoires, la propagation des incertitudes permet d'écrire que le carré de l'incertitude composée (fractionnaire) sur le paramètre, est la somme quadratique des incertitudes fractionnaires des différents termes (NF ENV13005, 1999): 


$$
\sigma_{\varphi}=\sqrt{\left(\frac{\partial \varphi}{\partial \mathrm{a}_{1}} \sigma_{\mathrm{a} 1}\right)^{2}+\left(\frac{\partial \varphi}{\partial \mathrm{a}_{2}} \sigma_{\mathrm{a} 2}\right)^{2}+\ldots .+\left(\frac{\partial \varphi}{\partial \mathrm{a}_{\mathrm{N}}} \sigma_{\mathrm{aN}}\right)^{2}}
$$

L'expression (5) représente en fait la propagation des incertitudes que l'on peut également écrire sous une forme condensée :

$$
\sigma_{\varphi}^{2}=\sum_{i=1}^{N}\left(\frac{\partial \varphi}{\partial a_{i}} \sigma_{a i}\right)^{2}
$$

L'exploitation des propriétés des sommes de carrés permet de simplifier les expressions des incertitudes en les bornant.

\section{4}

\section{Incertitudes de mesure}

\section{1.}

\section{Incertitude sur la mesure de déplacement}

L'erreur de mesure de chaque capteur de déplacement est fournie par le constructeur sous la forme d'un écart de linéarité (NF E11-062, 1985). Elle est de $0,1 \%$ pour une étendue de mesure de $100 \mathrm{~mm}$, soit une incertitude de $\pm 0,05 \mathrm{~mm}$.

La résolution de la chaîne d'acquisition est donnée par sa capacité à intégrer la mesure. Ici le déplacement est intégré sur 10000 points (pleine échelle). La résolution de la chaine est de $0,01 \mathrm{~mm}$, soit une incertitude sur la mesure de $\pm 0,005 \mathrm{~mm}$.

Finalement, l'incertitude de mesure d'un dépla cement est la somme des deux incertitudes évoquées précédemment, c'est-à-dire $\pm 0,055 \mathrm{~mm}$.

Les deux capteurs de déplacement sont fixés par l'intermédiaire d'une rotule sur le modèle. Dans un souci de simplification des calculs, l'incertitude incluite par l'usinage peut être négligée par rapport à l'incertitude liée au capteur de déplacement.

L'incertitude sur le déplacement au point d'application de la charge se déduit de l'équation (1). On obtient alors :

$$
\delta y=\delta y_{C 1}+\left|\delta y_{c 2}+\delta y_{c 1}\right| \times \frac{20}{45}
$$
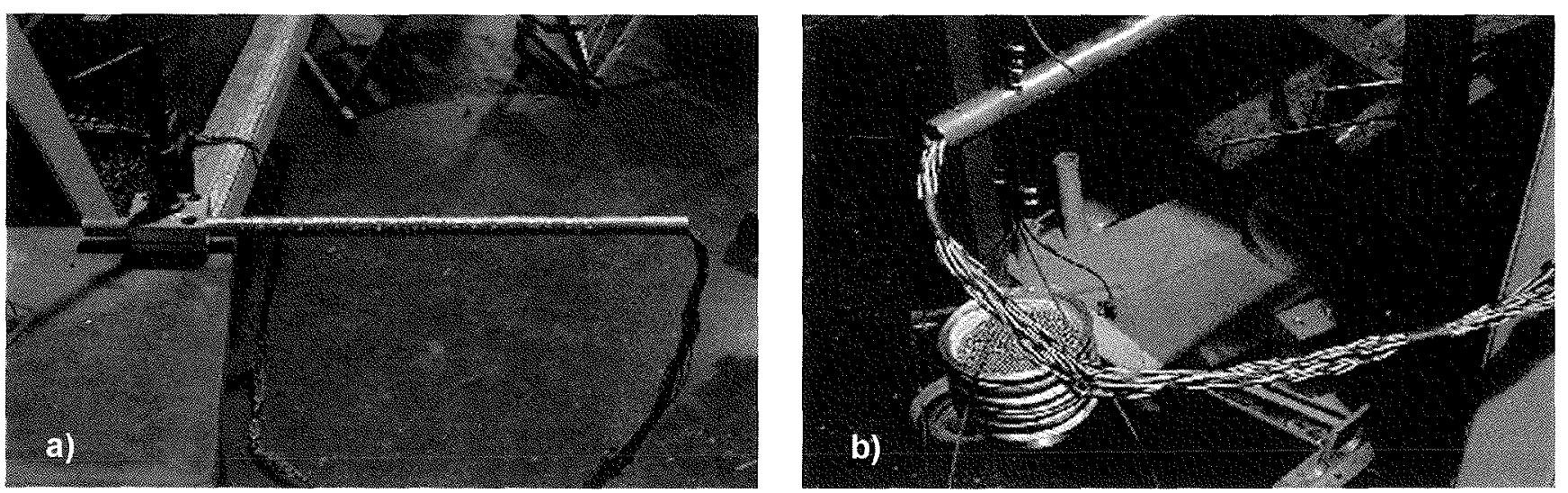

\section{Incertitude sur la mesure de force}

L'incertitude de mesure sur le capteur de force est donnée par le constructeur, comme pour le capteur de déplacement, sous la forme d'un écart de linéarité. Cet écart est de $0,15 \%$ sur l'étendue de mesure, ce qui représente une incertitude sur la mesure de la force de $3,75 \mathrm{~N}$, sachant que l'étendue de la mesure est de $5000 \mathrm{~N}$ (NF E11-062, 1985).

La chaîne d'acquisition intègre l'étendue de mesure de la force sur 10000 points. La résolution de cette dernière est donc de $0,5 \mathrm{~N}$, soit une incertitude de $\pm 0,25 \mathrm{~N}$.

L'incertitude de la mesure de force est la somme des incertitudes du capteur et de la chaîne d'acquisition, soit $\pm 4 \mathrm{~N}$.

\section{8.}

\section{Incertitude sur la mesure du moment}

On distingue trois sources d'incertitude lors de la mesure des moments de flexion dans le pieu : celles qui sont liées aux mesures $\delta \mathrm{Mm}$, au positionnement des jauges $\delta \mathrm{Mj}$ et à la mise en place du pieu $\delta \mathrm{Mp}$.

L'incertitude de mesure peut être déterminée (équation 8) à partir des données obtenues lors de l'étalonnage. Le montage réalisé, lors de cette opération, est de type (c console » (Fig. 5).

$$
\frac{\delta M m(z)_{i}}{M(z)_{i}}=\sqrt{\left(\frac{\delta E}{E}\right)^{2}+\left(\frac{\delta I}{I}\right)^{2}+\left(\frac{\delta B}{B}\right)^{2}+\left(\frac{\delta \Phi}{\Phi}\right)^{2}+\left(\frac{\delta k_{i}}{k_{i}}\right)^{2}+\left(\frac{\delta Q}{Q}\right)^{2}}
$$

La bibliographie fournit un module d'Young pour l'aluminium compris entre 74000 et $75000 \mathrm{MPa}$. L'incertitude sur le module pourra être prise égale à $\pm 500 \mathrm{MPa}$ ce qui donne un rapport $\delta \mathrm{E} / \mathrm{E}$ d'environ $\pm 0,7 \%$

L'incertitude sur le diamètre du tube est estimée à $1 / 10$ de millimètre soit, pour un diamètre du tube de $18 \mathrm{~mm}$, une incertitude $\delta \mathrm{B} / \mathrm{B}$ de $0,6 \%$.

L'incertitude sur le moment d'inertie peut être déterminée à partir du calcul du moment d'inertie d'un tube en effectuant une dérivée par rapport au diamètre extérieur B. 


$$
\frac{\delta I}{I}=\frac{4\left(B^{3}-b^{3}\right)}{B^{4}-b^{4}} \delta B
$$

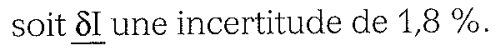

L'incertitude sur le type de jauge $\Phi$ ne dépend que de la tension d'alimentation du pont de jauges, soit $10 \mathrm{~V}$. La précision de l'alimentation est de $\pm 1 \mathrm{mV}$, soit une incertitude sur $\delta \Phi / \Phi$ de $\pm 0,01 \%$.

L'incertitude sur la linéarité des jauges $\mathrm{k}_{\mathrm{i}}$ peut être déterminée en étudiant leurs réponses à partir de la courbe de l'évolution du signal électrique en fonction de la charge appliquée lors de l'étalonnage $Q$ et peut être négligée au regard des résultats obtenus (Fig. 6).

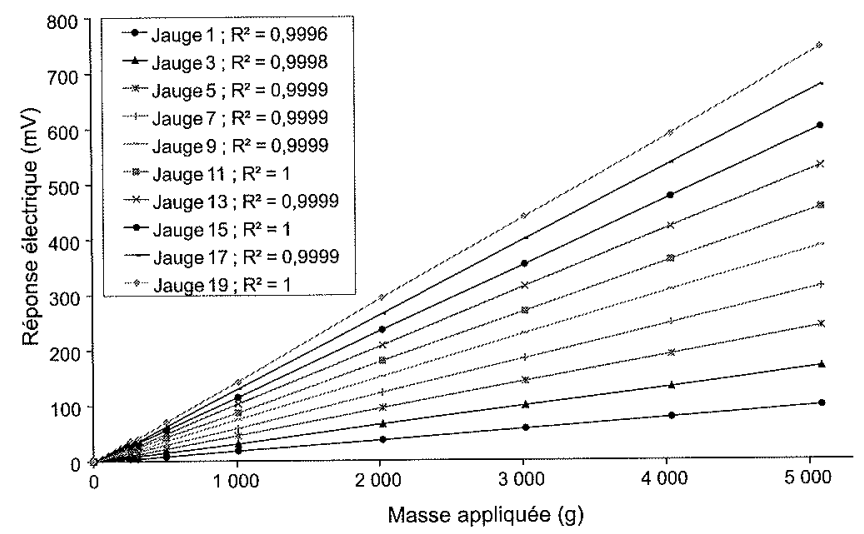

16. 6 Réponse des jauges en fonction de la masse appliquée (pour les numéros de jauges impaires).

Les masses utilisées pour appliquer Q sont calibrées. L'incertitude sur ces dernières est égale à la précision de la balance digitale, c'est-à-dire de $\pm 0,1 \mathrm{~g}$, soit pour la masse minimum appliquée lors de l'étalonnage (257 g) une incertitude $\delta$ Q/Q de $\pm 0,04 \%$.

L'incertitude $\delta \mathrm{Mm}(\mathrm{Z})_{i}$ est toujours majorée par la somme des incertitudes fractionnaire, donc $\delta \mathrm{Mm}(\mathrm{z})_{i}=3,1 \% \times \mathrm{M}(\mathrm{z})_{i}$.

L'incertitude sur le moment due à la position de la jauge peut être calculée à partir de la relation suivante, établie lors de l'étalonnage du pieu en console, avec A, la distance entre l'encastrement et le point d'application de la charge (Fig. 7) et $z_{i}$, la distance entre l'encastrement et la jauge $\mathrm{i}$ :

$$
\left|\frac{\delta M j(z)_{i}}{M(z)_{i}}\right| \leq\left|\frac{\delta A}{\left(A-z_{i}\right)}\right|+\left|\frac{\delta z_{i}}{\left(a-z_{i}\right)}\right|+\left|\frac{\delta Q}{Q}\right|
$$

L'entreprise qui a réalisé le collage des jauges assure le positionnement au 1/10 de millimètre près. La distance entre l'encastrement et le point d'application de la charge est connue à $\pm 0,5 \mathrm{~mm}$ (précision de mesure de la distance entre les deux points).

Dans la configuration d'étalonnage, où $\mathrm{A}=407 \mathrm{~mm}$, les distances $\mathrm{z}_{\mathrm{i}}$ entre l'encastrement et les jauges 1 et 20 sont respectivement de 22 et $307 \mathrm{~mm}$. L'incertitude sur le moment mesuré varie entre $\pm 0,6 \%$ et $\pm 0,2 \%$ pour la masse appliquée la plus faible (257 g).

Lors de la mise en place du pieu par battage, malgré toutes les précautions prises pour faire en sorte que la fibre neutre du pieu soit bien perpendiculaire au point d'application de la charge, il est possible qu'il y ait une légère rotation du pieu. Pour quantifier l'influence de cette rotation, on a modélisé le pieu avec le logiciel de calcul par éléments finis CESAR-LCPC (Rosquoët, 2004), en considérant un matériau élastique linéaire, avec comme conditions aux limites un encastrement en pied et une totale liberté en tête. Le calcul de la déformation à la surface permet de constater qu'une rotation de \pm 3 degrés du pieu par rapport au point d'application de la charge a une influence négligeable sur la déformation en surface et par conséquent sur la mesure du moment par l'intermédiaire de la jauge de déformation.

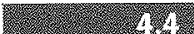

\section{Bilan}

Les incertitudes à prendre en compte sur la mesure de déplacements, de forces et de moments sont résumées dans le tableau I.

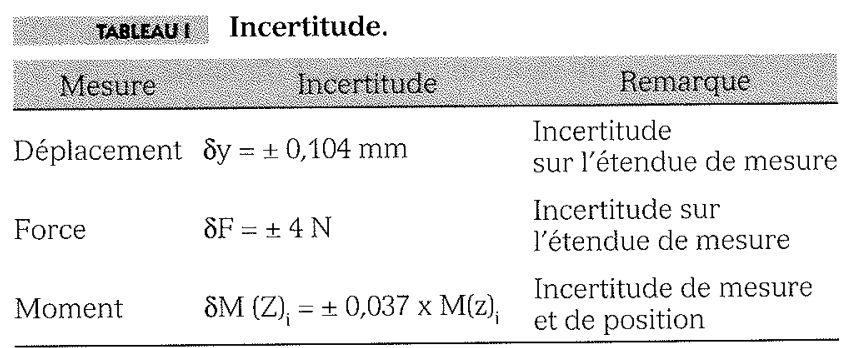

5

\section{Influence des incertitudes sur l'interpolation logarithmique}

On considère que le déplacement où le moment relatif et le nombre de cycles sont liés par une loi logarith-

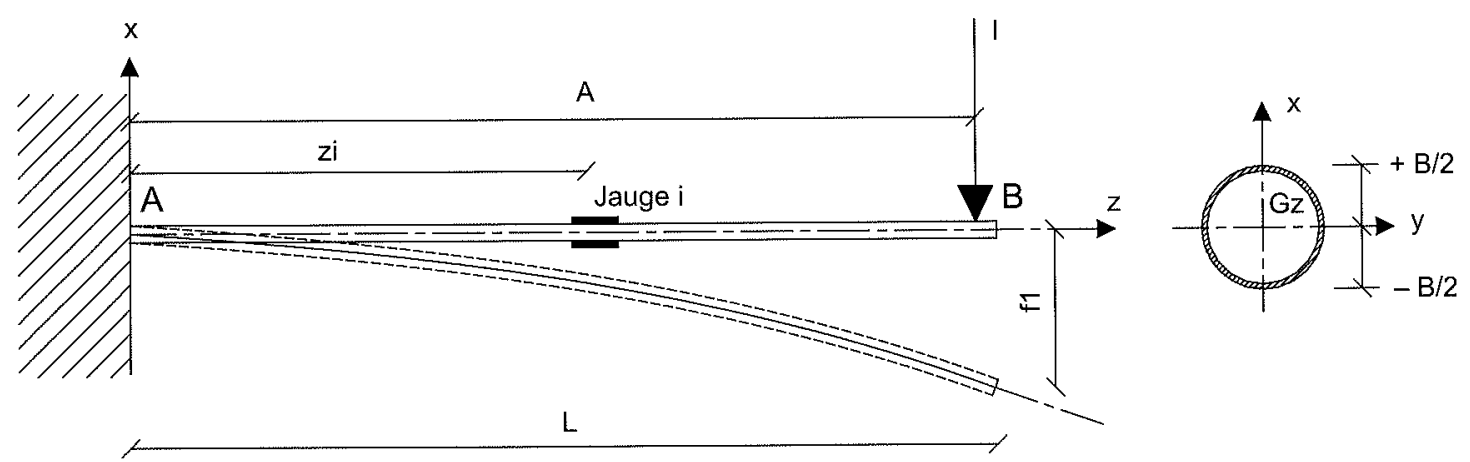


mique (Rosquoët, 2004). Les constantes ( a » et ( b » intervenant dans la fonction d'interpolation sont déterminées avec une certaine incertitude. On suppose que les incertitudes sur le moment et le déplacement relatif sont toutes de même amplitude et se repartissent suivant une loi de Gauss. On admet qu'il n'y a pas d'incertitude liée au nombre de cycles $n_{i}$.

On recherche une fonction logarithmique s'ajustant à l'ensemble des points $\left(n_{1}, C_{1}\right), \ldots,\left(n_{i}, C_{i}\right)$ de la forme:

$$
\mathrm{C}_{\mathrm{i}}=\mathrm{a}+\mathrm{bln}\left(\mathrm{n}_{\mathrm{i}}\right)
$$

On suppose que chaque mesure $C_{i}$ suit une distribution normale centrée sur la vraie valeur et dont la largeur est égale à l'écart-type $\sigma_{C}$. L'estimation de l'incertitude sur les constantes ( $a$ ) et ( $b$ b) se déduit alors de l'incertitude $\sigma_{\mathrm{Ci}}$ associée aux mesures de $\mathrm{C}_{\mathrm{i}}$ par la propagation des incertitudes, $\mathrm{N}$ étant le nombre total de cycles:

$$
\begin{aligned}
& \sigma_{\mathrm{b}}=\sigma_{\mathrm{Ci}}^{2} \sqrt{\frac{N}{\Delta}} ; \sigma_{a}=\sigma_{\mathrm{Ci}} \sqrt{\frac{\sum_{\mathrm{i}=1}^{N}\left(\ln \left(\mathrm{n}_{\mathrm{i}}\right)\right)^{2}}{\Delta}} \\
& \text { et } \Delta=N \sum_{\mathrm{i}=1}^{N}\left(\ln \left(\mathrm{n}_{\mathrm{i}}\right)\right)^{2}-\left(\sum_{\mathrm{i}=1}^{N} \ln \left(\mathrm{n}_{\mathrm{i}}\right)\right)^{2}
\end{aligned}
$$

\section{Influence des incertitudes sur l'analyse de l'effet des cycles}

Le chargement latéral cyclique est caractérisé par deux paramètres qui sont la charge maximum appliquée F et l'amplitude de la variation de charge DF. Pour un indice de densité du massif de sable de $86 \%$, sept essais ont été réalisés avec différentes valeurs de F et DF (repérés P32, P33, P36, P318, P344, P346, P347 sur les figures 8 et 9). Pour quantifier l'influence de la charge maximale et de l'amplitude sur le déplacement et le moment maximum, on a étudié leurs évolutions au cours des cycles. Afin de comparer les différents cas de charge entre eux, on s'est intéressé au déplacement et au moment maximum uniquement lorsque la charge maximum est appliquée durant les cycles; ils sont exprimés sous la forme de valeurs relatives par rapport au premier cycle, afin de les rendre adimensionnels (en les divisant par les valeurs mesurées au premier chargement).

En toute rigueur, le moment maximum doit être calculé à partir de la courbe d'interpolation du profil des moments en cherchant la profondeur à laquelle la dérivée s'annule. Cependant, la courbe des moments ne peut être interpolée que par un polynôme de degré au moins égal à 7 (Rosquoët, 2004) (éq. 13). Il est ainsi nécessaire, pour calculer le moment maximum, de résoudre une équation de degré 6 :

$$
M=a+b z+c z^{2}+d z^{3}+e z^{4}+f z^{5}+g z^{6}+h z^{7}
$$

La profondeur expérimentale correspondant au moment maximal déduit d'un tel calcul est proche de 2,4 m, c'est-à-dire de la position d'une des jauges de mesure effectuée sur le niveau de jauge à $2,4 \mathrm{~m}$ de profondeur et la valeur rigoureuse déduite mathématiquement reste inférieure à $1 \%$ (Tableau II). Par la suite, le moment maximal est déduit directement des données expérimentales (mesure du niveau de jauge) à une profondeur de $2,4 \mathrm{~m}$

Les évolutions des déplacements ou des moments relatifs suivent des relations logarithmiques (Figs. 8 et 9). Verdure et al. (2003) ont déjà proposé une expression de ce type à partir des résultats d'une première série d'essais. On constate tout d'abord une bonne répétitivité des essais. La figure 7 montre aussi que l'effet des cycles sur l'évolution du déplacement en tête est plus faible pour des cycles d'amplitude $\mathrm{DF}=150 \mathrm{~N}$ que pour les cycles d'amplitude de 300 à $600 \mathrm{~N}$ et l'inverse en ce qui concerne les moments (Fig. 9).

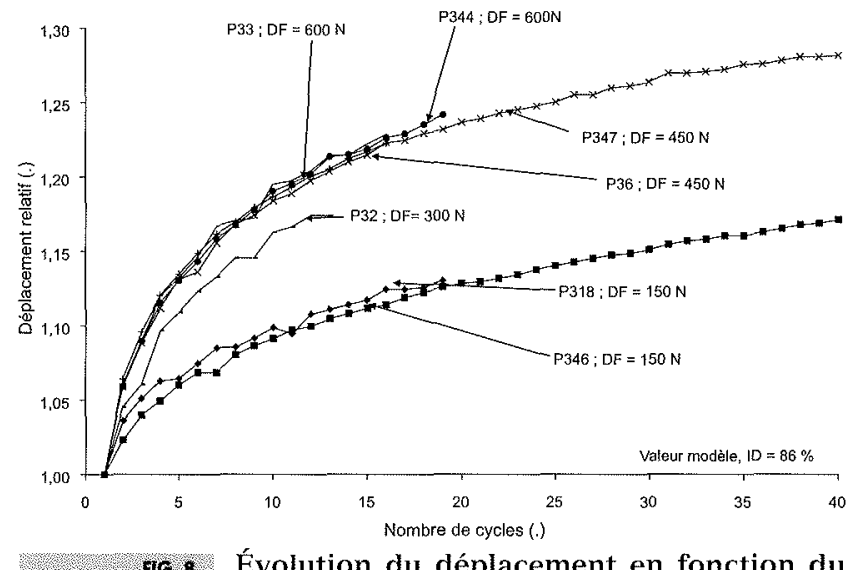

Évolution du déplacement en fonction du nombre de cycles $(F=600 \mathrm{~N}$, DF varie d'un essai à l'autre).

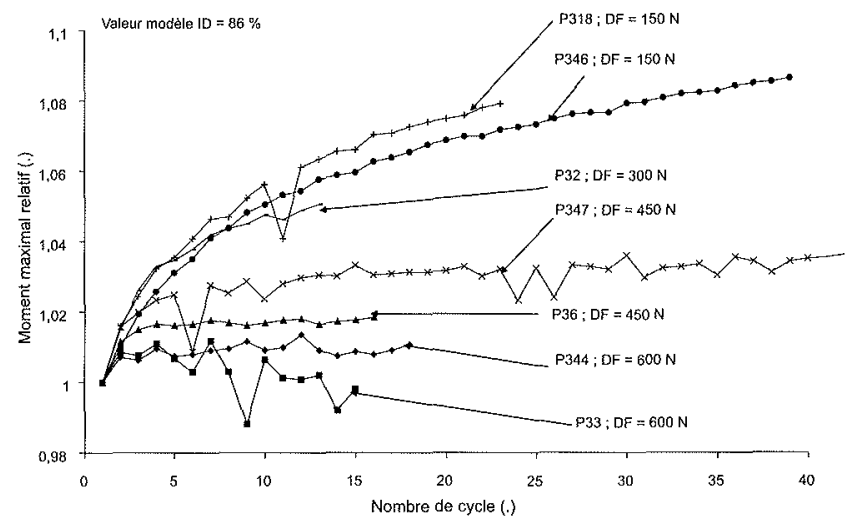

HG. 9 Évolution du moment relatif en fonction du nombre de cycles $(\mathrm{F}=600 \mathrm{~N}$, DF varie d'un

\begin{tabular}{|c|c|c|c|c|c|c|c|c|c|c|}
\hline & & & & & & & (c) & & (a) & 16 \\
\hline & Axy & Wath & r.xp & Math & Exp & Wath & $1 \times 10$ & Wath & $18 \times 10$ & Marn \\
\hline Profondeur $\mathrm{z}(\mathrm{m})$ & 2,4 & 2,5 & 2,4 & 2,58 & 2,4 & 2,66 & 2,4 & 2,79 & 2,4 & 2,78 \\
\hline Moment max. (kN.m) & 3048 & 3022,7 & 3128 & 3110 & 3154 & 3140 & 3193 & 3194 & 3201 & 3212 \\
\hline Écart sur le moment (\%) & & & & & & & & & & \\
\hline
\end{tabular}
essai à l'autre).

Taresui Comparaison entre le moment maximal expérimental (Exp.) et le moment maximal déduit de la courbe d'interpolation (Math.). 
Pour chaque cas de charge (F, DF), en supposant qu'il n'y a pas d'incertitude liée au programme d'asservissement du servo-verin, le déplacement relatif en fonction du nombre de cycles peut être interpolé par une fonction de type logarithme (éq. 11).

L'interpolation des courbes est réalisée par la méthode des moindres carrés. Pour tous les cas étudiés, les valeurs expérimentales sont proches de la loi choisie (coefficient de corrélation $R^{2}=0,98$ ). Cependant, le nombre de cycles réalisés au cours des essais ne permet pas de dire si la fonction d'interpolation choisie est bornée. Des essais réalisés récemment sur un sable moyennement dense $\left(\mathrm{I}_{\mathrm{D}}=50 \%\right)$ montrent, dans le cas d'un grand nombre de cycles, deux régimes de déplacement en tête : l'un avec une évolution de type logarithmique notable jusqu'à environ 100 cycles environ, l'autre plus faible, au-delà (Rakotonindriana et al., 2009).

Au vu de la figure 10, on remarque que le coefficient ( $(\mathrm{b})$ dépend de l'amplitude des cycles. Lorsque l'amplitude des cycles DF tend vers 0 , on est dans le cas d'un essai de fluage puisque la charge est constante et égale à $F$. Le déplacement induit par le fluage est négligeable (Rosquoët et al., 2007) ; par conséquent, le coefficient $(\mathrm{b})$ est proche de 0 .

Dans le cas de l'évolution du déplacement relatif, on constate que, même si l'incertitude sur le coefficient ( $\mathrm{b}$ ) n'est pas négligeable (Fig. 10, Tableau III), ( $(\mathrm{b})$ semble être croissant avec l'amplitude DF. Cette incertitude varie entre $12 \%$ pour une amplitude de $450 \mathrm{~N}$ et $38 \%$ pour l'amplitude de $150 \mathrm{~N}$ (plus le déplacement est faible, plus l'incertitude est importante). Ce résultat semble logique puisque l'incertitude sur la mesure est donnée par un écart de linéarité sur l'éten- due de mesure. Cependant, si l'on suit cette logique, la meilleure précision aurait correspondu à l'amplitude de $600 \mathrm{~N}$ (incertitude respective de 23 et $21 \%$ ), ce qui n'est pas vérifié. On remarque que le nombre de points d'interpolation (40 pour $450 \mathrm{~N}$, essai P347 contre 18 pour $600 \mathrm{~N}$, essai P36) a une influence sur les résultats du calcul. Plus la courbe d'interpolation est précise, plus l'incertitude de mesure a d'effet sur l'incertitude du coefficient ( $b$ ). Les mêmes remarques, hormis l'évolution selon DF, peuvent être formulées pour le calcul des incertitudes sur le coefficient ( $\mathrm{b}$ ) à partir de la mesure des moments (Fig. 11). Cependant, du fait d'une interpolation des courbes de moments de moins bonne qualité $\left(R^{2}=0,85\right)$, notamment pour le cas où les amplitudes sont importantes, les incertitudes sur le coefficient ( $\mathrm{b}$ ) peuvent dépasser $700 \%$, ce qui, d'un point de vue physique, n'a plus aucune signification. Par contre pour l'essai P346 où le coefficient de corrélation est proche de 1, l'incertitude est de $23 \%$. Sans surprise, plus le nombre de cycles est important, plus faible est l'incertitude sur l'identification de $($ b $b$. Cela corrobore, pour le nombre de cycles réalisés, l'hypothèse d'un ajustement logarithmique pour le déplacement relatif et le moment maximum relatif (Rosquoët, 2004).

\section{Application à un ouvrage prototype}

Le raisonnement présenté concerne les données obtenues sur des modèles réduits centrifugés. Pour acquérir des résultats sur l'ouvrage en vraie grandeur

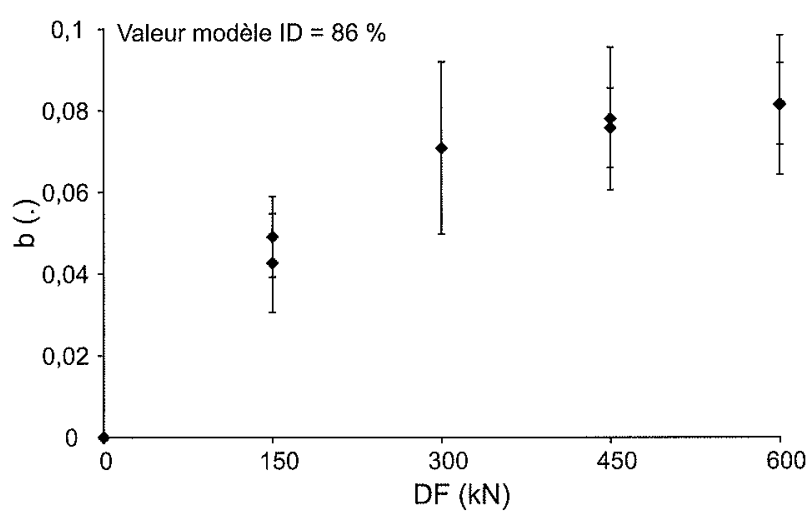

Évolution du coefficient $(a b$ ) en fonction de l'amplitude des cycles pour $\mathrm{F}=600 \mathrm{~N}$ : incertitudes sur les déplacements relatifs.

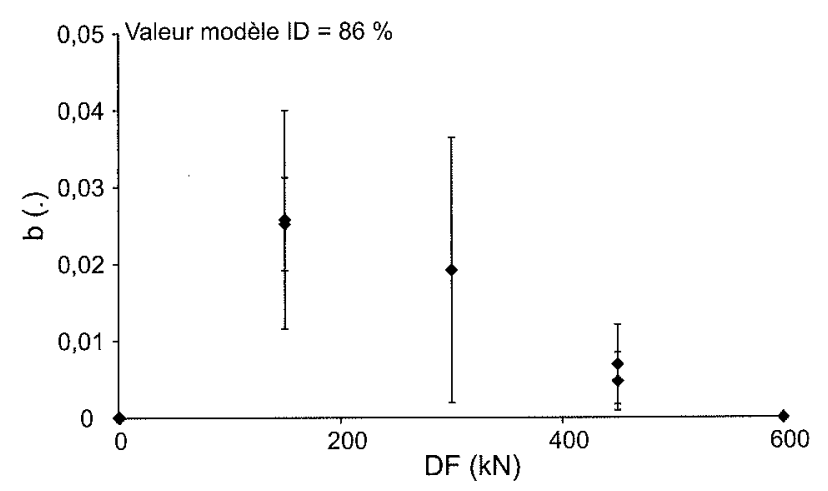

FG. 11 Évolution du coefficient “ $b$ ) en fonction de I'amplitude des cycles pour $\mathrm{F}=600 \mathrm{~N}$ : incertitudes sur les moments maximaux relatifs.

TABLEU in Estimation de l'incertitude sur la constante ( $b$ )).

\begin{tabular}{|c|c|c|c|c|c|c|c|c|}
\hline Essal & $\begin{array}{l}\text { Nombre } \\
\text { de cycles }\end{array}$ & (NI) & & (t) & 8 & & b) & 0 \\
\hline P33 & 14 & 960 & 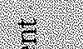 & 0,082 & 0,019 & & 0 & 1 \\
\hline P344 & 14 & 960 & $\vec{E}$ & 0,081 & 0,017 & 5 & 0 & 1 \\
\hline P36 & 18 & 720 & $8 \frac{7}{3}$ & 0,078 & 0,017 & $\frac{6}{6}$ & 0,0047 & 0,0038 \\
\hline P347 & 40 & 720 & $\frac{2}{2}$ & 0,075 & 0,01 & 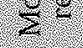 & 0,0069 & 0,0052 \\
\hline P32 & 15 & 480 & 8 & 0,071 & 0,021 & & 0,019 & 0,017 \\
\hline P318 & 25 & 240 & & 0,044 & 0,017 & & 0,026 & 0,014 \\
\hline P346 & 40 & 240 & & 0,049 & 0,01 & & 0,025 & 0,006 \\
\hline
\end{tabular}


(ou prototype), il faut appliquer les relations de similitude reliant le modèle réduit au prototype via un facteur d'échelle (Tableau IV).

De plus, l'accélération centrifuge dépend de la distance entre le point considéré du massif et l'axe de la centrifugeuse et varie avec le carré de la vitesse de rotation angulaire. Cette dernière est asservie en agissant sélectivement sur l'embrayage ou le frein, le signal de mesure étant fourni par un codeur optique de 6000 points (Corté et Garnier, 1984). Sur un palier de vitesse, la déviation maximale autorisée par rapport à la consigne est de $\pm 1 \%$ sur une durée de $24 \mathrm{~h}$.

TAbienu N Relations de similitude.

\begin{tabular}{lc}
\hline & Grandétr plysigue \\
\hline Longueur, déplacement & $\mathrm{L}^{*}=1 / \mathrm{n}$ \\
Accélération centrifuge et sismique & $\mathrm{g}^{*}=\mathrm{n}$ \\
Contrainte, pression & $\sigma^{*}=1$ \\
Déformation & $\varepsilon^{*}=1$ \\
Force & $\mathrm{G}^{*}=1 / \mathrm{n}^{2}$ \\
Masse & $1 / \mathrm{n}^{3}$ \\
Énergie & $1 / \mathrm{n}^{3}$ \\
Temps (dynamique) & $\mathrm{T}^{*}=1 / \mathrm{n}$ \\
Fréquence & $\mathrm{F}^{*}=\mathrm{n}$ \\
Vitesse, célérité des ondes & $\mathrm{V}^{*}=1$ \\
\hline
\end{tabular}

Par exemple, la force mesurée sur le modèle réduit est multipliée par $n^{2}$ pour le prototype, soit 1600 (échelle du pieu 1/40).

\section{Conclusion}

Les incertitudes sur les mesures, lors d'essais sur des modèles réduits centrifugés de pieux, sont de deux types: soit l'incertitude est liée à l'étendue de mesure (cas des capteurs de force et de déplacement), soit elle est proportionnelle à la mesure (moment). Les calculs d'incertitude s'appuient sur deux hypothèses :

- la mesure suit une loi normale (ou de Gauss);

- l'incertitude sur une mesure est égale à l'écart-type.

Les incertitudes de chaque appareil nous confortent dans ces choix.

Le calcul des incertitudes sur le coefficient $($ b $b$ ) de la fonction logarithmique montre que les incertitudes sur ce coefficient sont comprises entre 12 et $30 \%$, ce qui reste acceptable et doit permettre d'envisager une utilisation pratique des expressions proposées.

Cependant, on remarque qu'un grand nombre de points (cycles) engendre une meilleure précision sur l'interpolation, ce qui conforte le choix de la fonction logarithmique. Plus la courbe d'interpolation est précise, plus l'incertitude de mesure a d'effet sur l'incertitude concernant le coefficient $(\mathrm{b})$ ). Toutefois, il semble difficile de dissocier l'incertitude de mesure et l'approximation qui est due à l'interpolation.

\section{Bibliographie}

Corté J.-F., Garnier J. - La centrifugeuse du LCPC. Présentation des installations. Rapport des laboratoires série géotechnique, GT5, 1984, $18 \mathrm{p}$

Garnier J. - Modèles réduits en mécanique des sols. Les modèles réduits en génie civil. Colloque AUGC, 1995, p. 21-44.

NF E11-062 - Comparateurs électroniques, Capteurs de " déplacement linéaire » Méthodes d'essai. AFNOR/CSTB, 1985, $12 \mathrm{p}$.

NF ENV 13005 - Guide pour l'expression des incertitudes de mesure, 1999, 118 p.

Rakotonindriana J., Le Kouby A., Thorel L. Mestat P. - Comportement d'un pieu isolé soumis à un chargement cyclique horizontal. XVIIth ICSMGE, Alexandrie, 5-9 octobre 2009, Hamza, Shahhien, El-Mossallamy (eds). M : Upress, vol. 2, p. 1217-1220.
Remaud D. - Modélisation physique de pieux sous charges latérales : problème d'interprétation et de validation. Comptes rendus du prix jeune chercheur 99 $2^{e}$ congrès universitaire de Génie civil, AUGC, Poitiers, 6-7 mai 1999, p. 185192

Rosquoët F. - Pieux sous charge latérale cyclique. Thèse de doctorat, École centrale et université de Nantes, 2004, $-306 \mathrm{p}$.

Rosquoët F., Thorel L., Garnier J., Canepa Y. - Horizontal cyclic loading of piles installed in sand. Soils and Foundations, vol. 47, n 5, 2007, p. 821-832.

Taylor J. - An introduction to error analysis, 1982. University Science Books. (Incertitudes et analyse des erreurs dans les mesures physiques. Dunod (trad. fr.), $2000,316 \mathrm{p}$.
Thorel L., Rauet G., Garnier J., Murillo C., Gaudicheau P., Néel A., Favraud C. Mesures en macrogravité sur modèles réduits d'ouvrages géotechniques. Bulletin de liaison des laboratoires des ponts et chaussées, $n^{\circ} 272-273$, spécial Métrologie, 2008, p. 93-131.

Verdure L., Levacher D., Garnier J. - Effet des cycles sur le comportement d'un pieu isolé dans un sable dense sous chargement latéral. Revue Française de génie civil, 2003, p. 1185-1210. 